\title{
revista da FACULDADE DE DIREITO
}




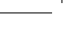




\section{UNIVERSIDADE FEDERAL DE MINAS GERAIS}

\section{revista da FACULDADE DE DIREITO}

ISSN: $0304-2340$

e-ISSN: $1984-1841$

$\overline{\text { Revista da Faculdade de Direito da UFMG | Belo Horizonte | Nova Fase-1962 | n.75 | pp. 1-566 | jul./dez. } 2019}$ 
(C) 2019 Revista da Faculdade de Direito da UFMG

Printed in Brazil

Qualquer parte desta publicação pode ser reproduzida, desde que citada a fonte.

As opiniões emitidas em artigos ou notas assinadas são de exclusiva responsabilidade dos respectivos autores.

Esta revista encontra-se disponível também em http://www.direito.ufmg.br/revista

DOI: 10.12818/P.0304-2340.2019v75

Pede-se permuta

Pídese canje

Man bittet um Austausch

We ask for Exchange

On demande l'échange

Si riquiere lo scambio

Esta publicação encontra-se indexada nas seguintes bases de dados: Ulrich's Periodical Directory, Bibliotheksservice-Zentrum Baden-Württemberg, ZBD: Zeitschriftendatenbank, e Latindex, dentre outras.

Revista da Faculdade de Direito da Universidade Federal de Minas Gerais. Nova Fase - 1962. - Belo Horizonte.

Títulos anteriores: Revista da Faculdade Livre de Direito do Estado de Minas Gerais, 1894 - 1928; Revista da Faculdade de Direito da Universidade de Minas Gerais, 1929 - 1948; Revista da Faculdade de Direito da Universidade Federal de Minas Gerais, 1949 - 1961; Revista da Faculdade de Direito da Universidade Federal de Minas Gerais - Nova Fase, 1962.

ISSN: $0304-2340$

E-ISSN: $1984-1841$

Periodicidade: semestral

1 - Direito - Periódicos - Faculdade de Direito da UFMG

CDU - 34 (05)

CDU - 340-05

Foto da Capa: Foca Lisboa - Painel de Jarbas Juarez, 1997 - Auditório da Faculdade de Direito da UFMC Projeto Gráfico, Formatação e Capa: Júnior Sena

Impressão: Imprensa Universitária da UFMG

Tiragem: 120 exemplares

Valor, nesta data, por unidade $\mathrm{R} \$ 30,00$

Os números atrasados da Revista da Faculdade poderão ser solicitados no endereço abaixo:

FACULDADE DE DIREITO DA UFMG

Revista da Faculdade

Av. João Pinheiro, 100 - 15o andar, sala 1507 / 1508

CEP 30130-180 - Belo Horizonte - MG - Brasil

Tel.: + $55313409-8651$

http://www.direito.ufmg.br/revista

revista@direito.ufmg.br 


\title{
REVISTA DA FACULDADE DE DIREITO UNIVERSIDADE FEDERAL DE MINAS GERAIS
}

(Fundada em 1894)

Palavras do Conselheiro Affonso Penna no lançamento da Revista da Faculdade de Direito: "Encetando a publicação de sua Revista, vem a Faculdade Livre de Ciências Jurídicas e Sociais da capital do Estado de Minas Gerais contribuir com o seu modesto auxílio para o estudo do Direito, pedra angular sobre que repousam as sociedades humanas."

\author{
REITORA DA UFMG \\ Profa. Dr ${ }^{a}$. Sandra Regina Goulart Almeida \\ VICE-REITOR DA UFMG \\ Prof. Dr. Alessandro Fernandes Moreira \\ DIRETOR DA FACULDADE DE DIREITO DA UFMG \\ Prof. Dr. Hermes Vilchez Guerrero \\ DIRETORA-EDITORA \\ Prof ${ }^{a}$. Dr ${ }^{a}$. Tereza Cristina Sorice Baracho Thibau \\ DIRETOR-ADJUNTO \\ Prof. Dr. Lucas Carlos Lima \\ ASSESSORA EDITORIAL \\ Rosali Ramos Diniz \\ ASSISTENTE EDITORIAL \\ Luísa Côrtes Grego \\ Flaviano Luiz Milagres Araújo \\ Sofia Neto Oliveira

\section{CONSELHO EDITORIAL}

Prof. Dr. Alejandro Montiel Alvarez (Universidade Federal do Rio Grande do Sul - Porto Alegre, MG - Brasil)

Prof. Dr. Alessandro Hirata (Faculdade de Direito da USP - Ribeirão Preto, SP - Brasil)

Prof. Dr. Andrew Drzemczewski (Conselho da Europa - Strasbourg, Alsace - France)

Prof. Dr. Antônio Augusto Cançado Trindade (Universidade de Brasília - Brasília, DF - Brasil)

Prof. Dr. Bjarne Melkevik (Université Laval - Quebéc, Quebéc - Canadá)

Prof ${ }^{a}$. Dr ${ }^{a}$. Daniela de Freitas Marques (Universidade Federal de Minas Gerais - Belo Horizonte, MG - Brasil)

Prof. Dr. Dean Spielmann (University of Luxembourg - Walferdange, Luxembourg - Luxembourg)

Prof ${ }^{a}$. Dr ${ }^{a}$. Elena Lisanyuk (State University of Saint Petersburg - Saint Petersburg, Northwestern - Russia)

Prof $f^{a}$ Dr ${ }^{a}$. Fabiana de Menezes Soares (Universidade Federal de Minas Gerais - Belo Horizonte, MG - Brasil)

Prof. Dr. Georges Abboud (Pontifícia Universidade Católica de São Paulo, São Paulo - Brasil)

Prof. Dr. Germain Ngoie Tshibambe (Université de Lubumbashi - Lubumbashi, Katanga - República Democrática do Congo)

Prof. Dr. Giovani Clark (Universidade Federal de Minas Gerais - Belo Horizonte, MG - Brasil)

Prof. Dr. Gustavo Silveira Siqueira (Universidade do Estado do Rio de Janeiro - Rio de Janeiro, RJ - Brasil)

Prof. Dr. Héctor Fix Zamúdio (Universidad Nacional Autónoma de México - Ciudad de Mexico, DF - Mexico)

Prof. Dr. Hugo Llanos Mansilla (Universidad Católica de Chile - Santiago - Chile)

Prof. Dr. Jean Paul Cabral Veiga da Rocha (Universidade de São Paulo - São Paulo, SP - Brasil)

Prof. Dr. João Maurício Leitão Adeodato (Universidade Federal de Pernambuco - Recife, PE - Brasil)

Prof ${ }^{a}$. Dr $r^{a}$. Juliana Neuenschwander Magalhães (Universidade Federal do Rio de Janeiro - Rio de Janeiro, RJ - Brasil)

Prof. Dr. Juan Ramón Capella Hernandez (Universidad de Barcelona - Barcelona, Cataluña - Espanha)

Prof ${ }^{a}$. Dr ${ }^{a}$. Lorena Ossio Bustillos (Max-Planck - Institute and German University of Administrative Sciences - Speyer,

Rheinland-Pfalz - Alemanha)

Prof. Dr. Mikhail Antonov (Law Faculty of National Research University "Higher School of Economics" - Saint

Petersburg, Northwestern - Russia)

Prof ${ }^{a}$. Dra. Miracy Barbosa de Sousa Gustin (Universidade Federal de Minas Gerais - Belo Horizonte, MG - Brasil)

Prof ${ }^{a}$. Dr ${ }^{a}$. Misabel de Abreu Machado Derzi (Universidade Federal de Minas Gerais - Belo Horizonte, MG - Brasil)

Revista da Faculdade de Direito da UFMG | Belo Horizonte | Nova Fase-1962 | n.75 | pp. 1 -566 | jul./dez. 2019 
Prof ${ }^{a}$. Dr ${ }^{a}$. Mônica Sette Lopes (Universidade Federal de Minas Gerais - Belo Horizonte, MG - Brasil)

Profa. Dra. Natasha Schmitt Cáccia Salinas (Fundação Getúlio Vargas - Rio de Janeiro, RJ - Brasil)

Prof. Dr. Nuno Manuel Morgadinho dos Santos Coelho (Universidade de São Paulo - Ribeirão Preto, SP - Brasil)

Prof. Dr. Paolo Palchetti (Université Paris 1 Panthéon - Sorbonne - França)

Prof. Dr. Pierre Issalys (Université Laval, Canadá - Quebéc, Quebéc - Canadá)

Prof. Dr. Ricardo Antônio Lucas Camargo (Universidade Federal do Rio Grande do Sul - Porto Alegre, RS - Brasil)

Prof. Dr. Roberto Luiz Silva (Universidade Federal de Minas Gerais - Belo Horizonte, MG - Brasil)

Profa. Dra. Suzy Elizabeth Cavalcante Koury (Centro Universitário do Estado do Pará - Belém, PA - Brasil)

Prof. Dr. Stephan Kirste (Universität Salzburg - Salzburg, Salzburg - Áustria)

Prof. Dr. Tércio Sampaio Ferraz Júnior (Universidade de São Paulo - São Paulo, SP - Brasil)

\section{CONSELHO DE PARECERISTAS AD HOC}

Profa. Dra. Adriane Medianeira Toaldo (Universidade Luterana do Brasil - Ulbra-RS)

Prof. Dr. Adriano Fernandes Ferreira - Universidade Federal da Amazonias - AM

Profa. Dra. Alessandra Marchioni (Universidade Federal de Alagoas - AL)

Profa. Dra. Alice Rocha da Silva (Centro Universitário de Brasília - DF)

Profa. Dra. Ana Claudia Pompeu Torezan Andreucci (Universidade Presbiteriana Mackenzie - SP)

Prof. Dr. Antônio Carlos Efing (Pontifícia Universidade Católica do Paraná - PR)

Prof. Dr. Antonio Pereira Gaio Júnior (Universidade Federal Rural do Rio de Janeiro - RJ)

Profa. Dra . Aparecida Luzia Alzira Zuin (Universidade Federal de Rondônia - RO)

Profa. Dra. Arianne Brito Cal Athias (Universidade Federal do Pará - PA)

Profa. Dra. Carla Noura Teixeira (Universidade da Amazônia - PA)

Prof. Dr. Carlos Nelson de Paula Konder (Pontifícia Universidade Católica do Rio de Janeiro - PUC RJ)

Prof. Dr. Cildo Giolo Júnior (Faculdade de Direito de Franca - SP)

Profa. Dra. Danielle Anne Pamplona (Pontifícia Universidade Católica do Paraná - PR)

Prof. Dr. Darci Guimarães Ribeiro (Universidade do Vale do Rio dos Sinos - RS

Prof. Dr. Delson Fernando Barcellos Xavier - Universidade Federal de Rondônia- RO

Prof. Dr. Dirley da Cunha Júnior (Universidade Federal da Bahia - BA)

Profa. Dra. Érika Mendes de Carvalho (Universidade Estadual de Maringá - PR)

Prof. Dr. Fernando César Costa Xavier (Universidade Federal de Roraima - RR)

Prof. Dr. Prof. Dr. Francisco Pedro Jucá (Faculdade Autônoma de Direito - SP)

Prof. Dr. Glauco Barreira Magalhães Filho (Universidade Federal do Ceará - CE)

Prof. Dr. Gustavo Filipe Barbosa Garcia (Faculdades Metropolitanas Unidas - SP e Centro Universitário do Distrito

Federal - Brasília)

Prof. Dr. Gustavo Noronha de Ávila (Universidade Estadual de Maringá PR)

Prof. Dr. Gustavo Silveira Siqueira (Universidade do Estado do Rio de Janeiro - RJ)

Prof. Dr. Iaci Pelaes dos Reis (Universidade Federal do Amapá - AP)

Prof. Dr. Igor Mauler Santiago (FGV, Universidade Federal Fluminense - UFF RJ)

Profa. Dra. Livia Mendes Moreira Miraglia (Universidade Federal de Minas Gerais - MG)

Profa. Dra. Marcia Carla Pereira Ribeiro (Universidade Federal do Paraná-PR)

Profa. Dra. Marcia Cristina Xavier de Souza (Universidade Federal do Rio de Janeiro - RJ)

Prof. Dr. Marcilio Toscano Franca Filho (Universidade Federal da Paraíba - PB)

Profa. Dra. Margareth Vetis Zaganelli (Universidade Federal do Espírito Santos - ES)

Profa. Dra. Maria Amália de Figueiredo Pereira Alvarenga (Universidade Estadual de São Paulo - SP)

Prof. Dr. Mateus de Oliveira Fornasier (Universidade Regional do Noroeste do Estado do Rio Grande do Sul -

Unijuí - RS)

Profa. Dra. Mônica Teresa Costa Sousa (Universidade Federal do Maranhão - MA)

Prof. Dr. Nilton César Antunes da Costa (Universidade Federal e Mato Grosso do Sul - MS)

Prof. Dr. Nuno Manuel Morgadinho dos Santos Coelho (Universidade de São Paulo - SP)

Prof. Dr. Paulo Eduardo Alves da Silva (Universidade de São Paulo - SP)

Prof. Dr. Pedro Moacyr Pérez da Silveira (Universidade Federal de Pelotas - RS)

Prof. Dr. Pedro Parini Marques de Lima (Universidade Federal de Pernambuco - PE)

Prof. Dr. Rafael da Silva Menezes (Universidade Federal do Amazonas - AM)

Prof. Dr. Raimundo Wilson Gama Raiol (Universidade Federal do Pará - PA)

Prof. Dr. Renato Poltronieri (Universidade Presbiteriana Mackenzie - SP)

Prof. Dr. Ricardo Antonio Lucas Camargo (Universidade Federal do Rio Grande do Sul - RS)

Prof. Dr. Rogério Montai de Lima (Universidade Federal de Rondônia - RO)

Prof. Dr. Saulo José Casali Bahia (Universidade Federal da Bahia - BA)

Prof. Dr. Sílvio César Arouck Gemaque (Universidade Municipal de São Caetano do Sul - RS)

Profa. Dra. Valéria Ribas do Nascimento (Universidade Federal de Santa Maria - RS)

Prof. Dr. Washington Carlos de Almeida (Universidade Presbiteriana Mackenzie - SP) 


\section{SUMÁRIO / CONTENTS}

\section{EDITORIAL}

$\ldots 11 \ldots$
EDITORIAL
Os artigos estão dispostos em ordem The articles are arranged in alphabetical alfabética dos nomes dos autores. order of author's names.

\section{ARTIGOS / ARTICLES}

A INFLUÊNCIA DO STF NA

REGULAMENTAÇÃO DO DEVER FUNDAMENTAL DE CONTRIBUIR COM A JUSTIÇA

Anselmo Laghi Laranja

Daury César Fabriz

PRISÃO E DESUMANIDADE NO BRASIL: UMA CRÍTICA BASEADA NA HISTÓRIA DO PRESENTE

Bruno Rotta Almeida

UNITED NATIONS ASSISTANCE

TO CONSTITUTION-MAKING

PROCESSES: PRE-CONSTITUENT AND

CONSTITUENT PHASES

Carina Barbosa Gouvêa

Jayme Benvenuto
... $17 \ldots$

$$
\text { ... } 43 \ldots
$$

Bruno Rotta Almeida

ASSISTÊNCIA DAS NAÇÕES UNIDAS PARA OS PROCESSOS DE CONSTITUTION-MAKING: FASES PRÉCONSTITUINTE E CONSTITUINTE

Carina Barbosa Gouvêa Jayme Benvenuto

A MULTIPROPRIEDADE IMOBILIÁRIA À THE REAL ESTATE MULTIPROPRIETY LUZ DA LEI 13.777/2018

UNDER THE LAW 13.777/2018

Cláudia Mara de Almeida Rabelo Viegas Rodolfo Pamplona Filho ... 91 ..

Cláudia Mara de Almeida Rabelo Viegas

INDICADORES EM TRÊS DIMENSÕES

PARA MONITORAMENTO

DE PROGRAMA DE JUSTIÇA

RESTAURATIVA

Daniela Carvalho Almeida da Costa ..119 ..

Daniela Carvalho Almeida da Costa

COMPLEMENTAR AS LEIS EXISTENTES

CONTRA ILEGAIS MONOPÓLIOS

E RESTRIÇÕES À ATIVIDADE

ECONÔMICA: A LEI CLAYTON DE 1914

Rodolfo Pamplona Filho

INDICATORS IN THREE DIMENSIONS FOR MONITORING OF RESTORATIVE

JUSTICE PROGRAM

Davi Monteiro Diniz

... $155 \ldots$

TO SUPPLEMENT THE EXISTING LAWS AGAINST UNLAWFUL MONOPOLIES AND RESTRAINTS OF TRADE: THE CLAYTON ACT OF 1914

A AUTORIZAÇÃO DE SERVIÇO PÚBLICO E A CONSTITUIÇÃO DE 1988

Davi Monteiro Diniz

THE AUTHORIZATION OF PUBLIC SERVICES AND THE 1988'S CONSTITUTION

Edilson Pereira Nobre Júnior ... $181 \ldots$ 
O DEBATE BIOÉTICO NUMA SOCIEDADE PÓS-MORALISTA, NA VISÃO DE LIPOVETSKY E JONAS

Edna Raquel Hogemann

IMPEACHMENT E O PRINCÍPIO DA LEGALIDADE COMO DIREITO HUMANO: CONTEÚDO, APLICABILIDADE E DEVER DE CONTROLE SOBRE O JUÍZO POLÍTICO

Eduardo Pitrez de Aguiar Corrêa

STATEHOOD, SELF-DETERMINATION AND INTERNATIONAL CRIMINAL JUSTICE. A FEW REMARKS

Giuseppe Nesi

A PRESENÇA DA IMAGINAÇÃO NA HERMENÊUTICA: UM ENCONTRO ENTRE IMMANUEL KANT E PAUL RICOEUR

Jaci Rene Costa Garcia

A OPINIÃO SOBRE O ARQUIPÉLAGO DE CHAGOS: A JURISDIÇÃO CONSULTIVA DA CORTE INTERNACIONAL DE JUSTIÇA E A NOÇÃO DE CONTROVÉRSIA
THE BIOETHIC DEBATE IN A POSTMORALIST SOCIETY IN THE VISION OF LIPOVETSKY AND JONAS ... $199 \ldots$

Edna Raquel Hogemann

IMPEACHMENT AND THE LEGALITY PRINCIPLE AS A HUMAN RIGHT: CONTENT, APPLICABILITY AND JUDICIAL REVIEW

... 223 ... Eduardo Pitrez de Aguiar Corrêa STATEHOOD, AUTODETERMINAÇÃO E JUSTIÇA PENAL INTERNACIONAL: ALGUMAS CONSIDERAÇÕES.

Giuseppe Nesi

THE PRESENCE OF IMAGINATION IN HERMENEUTICS: A MEETING BETWEEN IMMANUEL KANT AND PAUL RICOEUR ... 263... Jaci Rene Costa Garcia THE OPINION ON THE CHAGOS ARCHIPELAGO: THE ADVISORY JURISDICTION OF THE INTERNATIONAL COURT OF JUSTICE AND THE NOTION OF DISPUTE

Lucas Carlos Lima ... 281...

Lucas Carlos Lima

A DIFUSÃO DE SISTEMAS DE VIDEOVIGILÂNCIA NA URBE CONTEMPORÂNEA: UM ESTUDO INSPIRADO EM ARGOS PANOPTES, CÉREBROS ELETRÔNICOS E SUAS CONEXÕES COM A LIBERDADE E A IGUALDADE

Marcos Catalan ...303...

THE SPREAD OF VIDEOSURVAILLANCE SYSTEMS IN CONTEMPORARY CITIES: A STUDY INSPIRED BY ARGOS PANOPTES, ELETRONIC BRAINS AND ITS CONNECTIONS TO LIBERTY AND EQUALITY. Marcos Catalan

DEMOCRACIA DE GÊNERO E SEUS DASAFIOS: COMO AS AÇÕES AFIRMATIVAS PARA PARTICIPAÇÃO FEMININA NA POLÍTICA DEVEM SER APRIMORADAS

Marilda de Paula Silveira ...323... Marilda de Paula Silveira RIFLESSIONI SUL CONTROVERSO REATO DI TORTURA

SOME CONSIDERATIONS ABOUT THE CONTROVERSIAL CRIME OF TORTURE

Maristella Amisano Federica Giraldi ... 349..
Maristella Amisano Federica Giraldi 
LES INSTRUMENTS NON CONVENTIONNELS DE LA COMMISSION DU DROIT INTERNATIONAL DES NATIONS UNIES: SUCCES OU ECHEC DE LA CODIFICATION?

Maurizio Arcari

PARCERIAS ENTRE ESTADO E ORGANIZAÇÕES DA SOCIEDADE CIVIL: ANÁLISE DO SEU ESPAÇO REGULATÓRIO

Natasha Schmitt Caccia Salinas

THE IMPACT OF THE INTERAMERICAN COURT OF HUMAN RIGHTS JURISPRUDENCE IN THE INTERNATIONAL COURT OF JUSTICE CASE LAW

Paula Wojcikiewicz Almeida

POR UM ACESSO QUALITATIVO À JUSTIÇA - O PERFIL DA LITIGÂNCIA NOS JUIZADOS ESPECIAIS CÍVEIS

Paulo Eduardo Alves da Silva ... $443 \ldots$

Paulo Eduardo Alves da Silva

DA UNIÃO À FORTALEZA OU COMO A RELAÇÃO DE DEPENDÊNCIA ENTRE MIGRAÇÃO E INTEGRAÇÃO PODEM FAZER DESAPARECER A IDEIA DE UNIÃO EUROPEIA

... $419 \ldots$

Paula Wojcikiewicz Almeida

SUBSTANTIAL ACCESS TO JUSTICE - THE NATURE OF DISPUTES AND LITIGANTS ON SMALL CLAIMS COURTS IN BRAZIL

FROM UNION TO FORTRESS OR HOW THE RELATION OF DEPENDENCE BETWEEN MIGRATION AND INTEGRATION CAN PROVOQUE THE ABOLITION OF EUROPEAN UNION IDEALS

Paulo Potiara de Alcântara Veloso

REFLEXÕES ACERCA DA EXECUTIVIDADE DAS SENTENÇAS DE IMPROCEDÊNCIA EM AÇÕES DECLARATÓRIAS NEGATIVAS

Rosalina Moitta Pinto da Costa

MIXED AND HYBRID SYSTEMS OF JUSTICE AND THE DEVELOPMENT OF THE ADVERSARIAL PARADIGM: EUROPEAN LAW, INQUISITORIAL PROCESSES AND THE DEVELOPMENT OF COMMUNITY JUSTICE IN THE COMMON LAW STATES

... 467 ... Paulo Potiara de Alcântara Veloso

REFLECTIONS ON THE EXECUTIVITY OF REFUSAL JUDGMENTS IN NEGATIVE DECLARATORY ACTIONS ... $487 \ldots$

Rosalina Moitta Pinto da Costa MIXED AND HYBRID SYSTEMS OF JUSTICE AND THE DEVELOPMENT OF THE ADVERSARIAL PARADIGM: EUROPEAN LAW, INQUISITORIAL PROCESSES AND THE DEVELOPMENT OF COMMUNITY JUSTICE IN THE COMMON LAW STATES

Tyrone Kirchengast ...513 ... 


\section{MEMÓRIA HISTÓRICA / HISTORICAL MEMORY}

DISCURSO DE POSSE NA DIREÇÃO DA FACULDADE DE DIREITO DA UFMG
INAUGURAL ADRESS AS HEADMASTER OF FACULDADE DE DIREITO DA UFMG

Hermes Vilchez Guerrero ...539 ...

Hermes Vilchez Guerrero

SEMINARO ALL'UNIVERSITÀ DELLA CALABRIA - I

I CORSI GIURIDICI IN BRASILE

Sheila Jorge Selim de Sales

SEMINARO ALL'UNIVERSITÀ DELLA

CALABRIA - II

APPUNTI SUL PROGRAMMA DI

STUDIO E PROGRAMMA DELLA LEZIONE

Sheila Jorge Selim de Sales
...547 ...

...553 ... Sheila Jorge Selim de Sales

CONFERENCE IN THE UNIVERSITY

OF CALABRIA - I

LAW COURSES IN BRAZIL

Sheila Jorge Selim de Sales

CONFERENCE IN THE UNIVERSITY OF CALABRIA - II COMMENTS ON THE STUDY PROGRAM AND THE LECTIONS PLAN 